\title{
Avoiding entanglement loss when two-qubit quantum gates are controlled by electronic excitation
}

\author{
R Rodriquez ${ }^{1}$, A J Fisher, P T Greenland and A M Stoneham \\ Department of Physics and Astronomy, University College, London WC1E 6BT, UK
}

Received 21 August 2003

Published 8 April 2004

Online at stacks.iop.org/JPhysCM/16/2757

DOI: 10.1088/0953-8984/16/16/001

\begin{abstract}
A solid-state two-qubit quantum gate was recently proposed that might be made in a silicon fabrication plant in the near future. In this class of device, entanglement between two quantum bits is controlled by a change from a largely unentangled ground electronic state to an excited state in which useful entanglement can be produced. Such gates have potential advantages, both because they exploit known solid-state behaviour and they separate the storage and manipulation of quantum information. It is important that the excitation step does not create decoherence. We analyse a type of gate proposed before, in which the excitation involves a control electron that interacts with the qubit spins in the excited state. The dynamics of an idealized (but fairly general) gate of this type show that it can be operated to produce a standard two-qubit entangling state.
\end{abstract}

\section{Introduction}

Quantum computers use quantum logic gates (DiVincenzo 1995, Vedral and Plenio 1998), which manipulate quantum bits—qubits. Much study has shown that a universal set of quantum gates must include a two-qubit gate which can produce entanglement (Barenco et al 1995, Beckmann et al 1996). In order to do this, its qubits must interact with each other. This, of course, is a problem; in order to preserve coherence it is desirable to isolate the qubits from each other, and from the world, but in order to produce the entanglement necessary for a universal gate they must interact. Quantum computers based on ion traps (Jonathan et al 2000, Cirac and Zoller 1995) solve the problem by using special protocols to bring about interaction between otherwise almost perfectly isolated ions, but many solid-state devices rely on an ability to control the direct interaction between the qubits (Loss and DiVincenzo 1998, Golovach and Loss 2002). Often, this means that engineered intervention is required to set the naturally occurring interaction between the qubits to zero.

1 Present address: Centre for Quantum Computation, Department of Applied Mathematics and Theoretical Physics (DAMTP), University of Cambridge, Wilberforce Road, Cambridge CB3 OWA, UK. 
Quantum information processors will need to be integrated with classical digital microelectronics. The ideal quantum information processor would be one which could be manufactured in a standard fabrication facility of the type planned for the near future. No such quantum information processor has been demonstrated, but one recent proposal (Stoneham et al 2003), based on the change of entanglement controlled by electronic excitation, may lead to such a development. Our paper addresses a specific, but generic, aspect of such gates and, in particular, we show from an analysis of their dynamics that idealized gates of the type discussed by Stoneham et al can, in principle, be operated to produce standard two-qubit entangling gates, with negligible loss of quantum information to the control electron. This paper expands some of the details which are important for a processor of this type.

The unusual feature of our scheme is that we use 'control' electrons to manipulate our qubits. By suitably modifying the excitation state of the control electron we aim to modify the electron-electron interaction between control and qubit electrons to produce two-qubit entanglement. However, it is important that after it has done its job, the control electron is not only in a state which does not interact with the qubits, but also is not entangled with them. We show here that this is possible.

In particular we consider an idealization of the actual solid-state system that might be used in such a quantum gate. Our ideal system contains just three particles; we assume that two qubits are encoded by the spin states of two of them. We further assume that there is no interaction between the particles when they are all in their ground states, but that if one is in an excited state, it can interact with the other two. Thus, the gate protocol requires that we control the excitation and de-excitation of one of the particles - the control particle-so as to produce the required entanglement between the other two, in such a way that the control particle is ultimately returned to its unexcited state and is not entangled with the particles encoding the qubits. In our previous publication (Stoneham et al 2003), we described a possible implementation of this scheme in Si. In that case the qubit spins were envisaged to belong to deep donors in $\mathrm{Si}$, and the third (communicating) particle was an electron that could be excited into a delocalized 'molecular' state connecting both donors. Here we leave aside the details of the specific implementation and concentrate on the dynamics of the two-qubit gate under the action of a rather generic Hamiltonian. Thus, in this work we ignore many of the complications which will occur in practice, and focus on one important aspect of the quantum gate. What we describe here-or something similar - is necessary for the successful operation of the gate; it is certainly not sufficient. We do not discuss the implementation of single-qubit operations in this paper, but note that this may (in principle) be done using previously demonstrated techniques (Charnock and Kennedy 2001, Jelezko et al 2002).

\section{The three spin system}

In this section we describe the effective Hamiltonian which controls the interaction between the qubits. As we shall see, an important feature of our gate is that the qubit-qubit interactions are mediated by naturally occurring features of the system. External control is provided by laser pulses, but since these do not interact directly with the qubits, an important source of engineering noise is avoided.

\subsection{The spin basis}

Our system consists of three particles A, B and C in a magnetic field B. A and B are always in their ground electronic state, where it is assumed that they are decoupled from the environment. Their spin states encode the two qubits. $\mathrm{C}$ is the control particle. It is assumed that in its ground 


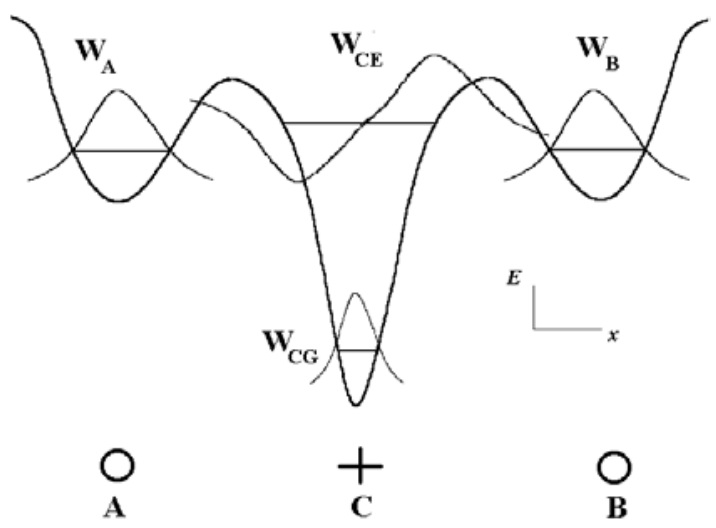

Figure 1. The quantum gate. The qubits are encoded in the spins of defects A and B with spatial wavefunctions $W_{\mathrm{A}}$ and $W_{\mathrm{B}}$. Laser excitation of the control atom $\mathrm{C}$ from its ground state, with wavefunction $W_{\mathrm{CG}}$ to the excited state $W_{\mathrm{CE}}$, controls the qubit interactions.

electronic state $\mathrm{C}$ is also decoupled from the environment (and from $\mathrm{A}$ and $\mathrm{B}$ ), but that $\mathrm{C}$ also has an excited electronic state which couples to A and B (and the environment). The configuration is sketched in figure 1 .

The wavefunction of this system has both spatial and spin components. For the three particles there are $2^{3}=8$ spin states in the electronic ground state, and $2^{3}$ spin states in the electronic excited state, a total of 16 states. We neglect orbital excitation of the qubit spins A and B. In order to proceed we construct a basis set. We are interested in the time development of the spins, and so shall find it convenient to use the conventional representation of $|0\rangle$ as spin up, and $|1\rangle$ as spin down, and write the state of the three particles A, B and C as $\psi_{\mathrm{A}} \psi_{\mathrm{B}} \psi_{\mathrm{C}}^{\mathrm{s}}|i j k\rangle$, where $i, j, k=0,1$ and the ordering is significant, so that $i$ represents the spin state of A, $j$ the spin state of B and $k$ the spin state of $\mathrm{C}$. The wavefunctions $\psi_{\mathrm{A}}, \psi_{\mathrm{B}}$ and $\psi_{\mathrm{C}}^{\mathrm{s}}$ represent the spatial part of the wavefunction for each of the particles; $\psi_{\mathrm{C}}^{\mathrm{s}}$ carries an extra label $\mathrm{s}$ to characterize the ground state of $\mathrm{C}(\mathrm{s}=\mathrm{g})$ or the excited state $(\mathrm{s}=\mathrm{e})$. Thus, our basis is

$$
\mathbf{b}=\left[\begin{array}{l}
\mathbf{v}_{\mathrm{g}} \\
\mathbf{v}_{\mathrm{e}}
\end{array}\right]
$$

with

$$
\mathbf{v}_{\mathrm{s}}=\psi_{\mathrm{A}} \psi_{\mathrm{B}} \psi_{\mathrm{C}}^{\mathrm{s}}[|000\rangle,|001\rangle, \ldots,|r\rangle, \ldots,|110\rangle,|111\rangle]^{\mathrm{T}} .
$$

Since we are almost always only interested in the spin wavefunctions we shall usually suppress the spatial part, and write

$$
\mathbf{v}_{\mathrm{s}}=[|000\rangle,|001\rangle, \ldots,|r\rangle, \ldots,|110\rangle,|111\rangle]_{\mathrm{s}}^{\mathrm{T}} .
$$

\subsection{The interaction Hamiltonian}

We assume that the major interaction between the three electrons in the excited state is exchange, and that this can be modelled with an effective Heisenberg interaction (Herring and Flicker 1964). We use units in which $\hbar=1$. Our Hamiltonian $H$ is therefore

$$
\begin{aligned}
H=|\mathrm{g}\rangle\left\{B_{\mathrm{A}} \sigma_{\mathrm{A} z}\right. & \left.+B_{\mathrm{B}} \sigma_{\mathrm{B} z}+B_{\mathrm{C}}^{0} \sigma_{\mathrm{C} z}\right\}\langle\mathrm{g}| \\
& +|\mathrm{e}\rangle\left\{J_{\mathrm{A}} \sigma_{\mathrm{A}} \cdot \sigma_{\mathrm{C}}+J_{\mathrm{B}} \sigma_{\mathrm{B}} \cdot \sigma_{\mathrm{C}}+B_{\mathrm{A}} \sigma_{\mathrm{A} z}+B_{\mathrm{B}} \sigma_{\mathrm{B} z}+B_{\mathrm{C}} \sigma_{\mathrm{C} z}+\epsilon\right\}\langle\mathrm{e}| \\
& +|\mathrm{e}\rangle V(t) \cos (\omega t+\phi)\langle\mathrm{g}|+| \mathrm{g}\rangle V(t) \cos (\omega t+\phi)\langle\mathrm{e}| .
\end{aligned}
$$


We have quantized along the magnetic field $\mathbf{B}$ and defined

$$
B_{\mathrm{K}}=-|\mathbf{B}| \mu_{\mathrm{K}} \quad(\mathrm{K}=\mathrm{A}, \mathrm{B}, \mathrm{C}) ; \quad B_{\mathrm{C}}^{0}=-|\mathbf{B}| \mu_{\mathrm{C}}^{0}
$$

where $\mu_{\mathrm{A}}$ and $\mu_{\mathrm{B}}$ are the magnetic moments associated with particles $\mathrm{A}$ and $\mathrm{B}$ (which are always in their ground states), $\mu_{\mathrm{C}}^{0}$ is the magnetic moment of particle $\mathrm{C}$ in its ground state, and $\mu_{\mathrm{C}}$ the magnetic moment of particle $\mathrm{C}$ in its excited state. Note that, if the magnetic moments $\mu_{\mathrm{K}}$ are different, $B_{\mathrm{A}}, B_{\mathrm{B}}$ and $B_{\mathrm{C}}$ as defined in equation (5) may differ even when the external magnetic field is uniform. We have assumed that when all three particles $\mathrm{A}, \mathrm{B}, \mathrm{C}$ are in their ground states there is negligible interaction between them, but that when $\mathrm{C}$ is in its excited state, which of course has a larger spatial extent, the effective exchange interactions between $\mathrm{A}$ and $\mathrm{C}$ and $\mathrm{B}$ and $\mathrm{C}$ have strengths $J_{\mathrm{A}}$ and $J_{\mathrm{B}}$, respectively. The exchange is modelled as Heisenberg $(\sigma \cdot \sigma)$ interactions. Although in $\mathrm{Si}$ the true exchange is complicated by the indirect nature of the bandgap, and consequent intervalley interference and anisotropic effective mass effects, Andres et al (1981) and Koiller et al (2002a, 2002b) show that the characteristic strength of the exchange interaction between defects can be related to the 'hydrogenic' value (Slater 1963, Herring and Flicker 1964), and this is what we assume. Thus, as we show in figure 2, there is a range of separations for which exchange between the ground states of $\mathrm{A}, \mathrm{B}$ and $\mathrm{C}$ is negligible, and for which exchange between the excited state of $\mathrm{C}$ and $\mathrm{A}$ and $\mathrm{C}$ and $\mathrm{B}$ is usefully large.

Furthermore the ground $(|\mathrm{g}\rangle)$ and excited $(|\mathrm{e}\rangle)$ states are coupled by the interaction $V(t) \cos (\omega t+\phi)$ as is appropriate for pulsed laser excitation of particle $\mathrm{C}$ in the semiclassical approximation. This laser interaction, in conjunction with the exchange in the excited state, is what controls the qubits. The excited state excitation energy is $\epsilon$. In the solid-state implementations we envisage this can be $\sim 1 \mathrm{eV}$, certainly less than the Si bandgap, so the energy scale for the gate operation can be large. The lifetime of the upper state is, of course, important for the operation of the gate. Although we do not have accurate values for this lifetime, values of $\sim \mu$ s are typical for such states (Stoneham 1975) (and consistent with values derived by scaling hydrogenic transition rates). We discuss below how the gate operation depends upon the upper state lifetime, and find that there are circumstances under which lifetimes considerably shorter than microseconds can still give acceptable gate performance.

The energy scale for the exchange splitting in the excited state, which, as we shall see below, determines the laser pulse length, is set by $J_{\mathrm{A}}, J_{\mathrm{B}}$. We envisage values for this effective interaction of the order of $1-10 \mathrm{GHz}$. The control-qubit separation required to achieve this can be estimated from figure 2. We find values of about 13-17 nm. (At this separation the magnetic dipole-dipole interaction between the active electrons is $\sim 10 \mathrm{peV}$, and we ignore it.) Further details of exchange strengths in Si can be found in Herring and Flicker (1964), Cullis and Marko (1970), Stoneham (1975), Andres et al (1981), Koiller et al (2002a, 2002b).

As we have written it, the first term in equation (4) represents the ground state part of the Hamiltonian, the second term the excited state part, and the last term the coupling between them.

With the basis set described in equation (1), the Hamiltonian can be written

$$
H=\left[\begin{array}{cc}
\mathbf{H}^{\mathrm{g}} & \mathbf{L}(t) \\
\mathbf{L}^{\dagger}(t) & \epsilon+\mathbf{H}^{\mathrm{e}}
\end{array}\right]
$$

Here $\mathbf{H}^{\mathrm{g}}$ is an $8 \times 8$ matrix describing the interactions in the ground state. It is diagonal, and its $r, r$ th element is the magnetic energy of the state $|r\rangle$, which can be written as

$$
\mathbf{H}_{r r}^{\mathrm{g}}=(-1)^{i} B_{\mathrm{A}}+(-1)^{j} B_{\mathrm{B}}+(-1)^{k} B_{\mathrm{C}}^{0}
$$

where $|r\rangle$ has the binary representation

$$
|r\rangle=|i j k\rangle \text {. }
$$




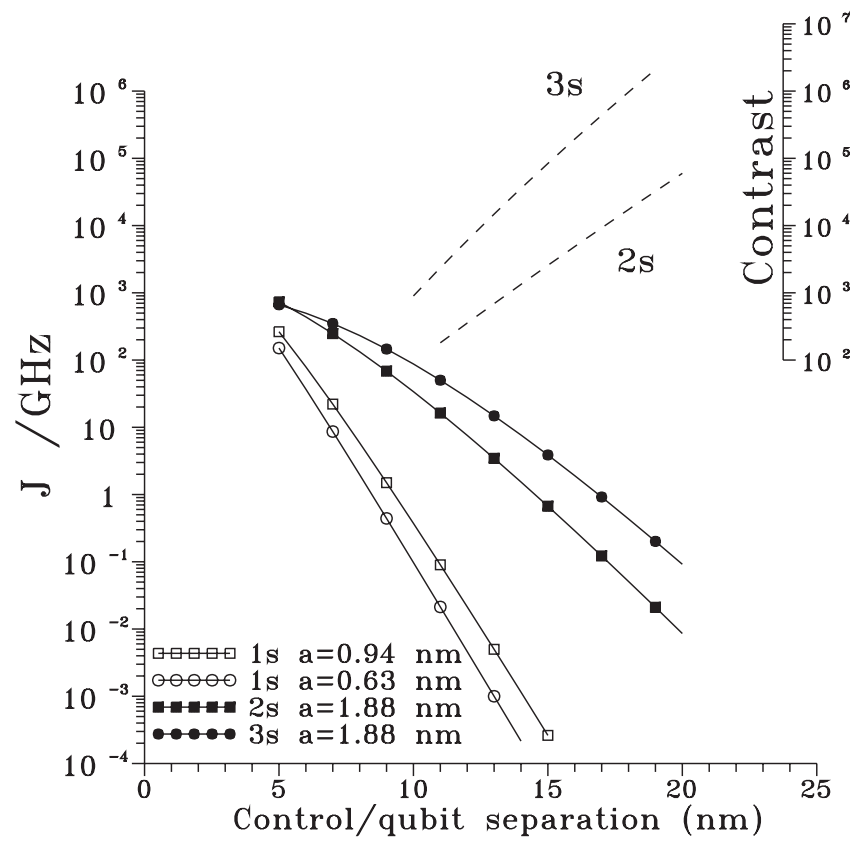

Figure 2. Effective mass theory estimate of the exchange interaction strength $J(\mathrm{GHz})$ as a function of separation between qubit and control atoms. The exchange is calculated numerically (Fernández Rico et al 1997, Alvarez Collado et al 1989) using $n$ s-type Slater orbitals (Slater 1963) of the form $r^{n-1} \exp (-r / a)$ with effective mass and dielectric constant appropriate to electrons in Si. The qubit is assumed to be in a 1s orbital with $a=1.28 \mathrm{~nm}$, as appropriate for the binding energy of the ground state of $\mathrm{Si}: \mathrm{Bi}$. Its exchange interaction with both the ground and excited states of the control atom are shown, for two cases whose parameters are representative: first for a control atom whose ground state is represented by a 1s Slater with $a=0.94 \mathrm{~nm}$ (open squares) and whose excited state is represented by a 2 s Slater with $a=1.88 \mathrm{~nm}$ (filled squares), and secondly for a control atom whose ground state is represented by a 1s Slater with $a=0.63 \mathrm{~nm}$ (open circles) and whose excited state is represented by a $3 \mathrm{~s}$ Slater with $a=1.88 \mathrm{~nm}$ (filled circles). Also shown, as dashed curves, are the excited/ground state contrasts, the ratio of the exchange in the excited state to exchange in the ground state for the two cases (dashed curves and right-hand scale). Although these calculations do not reflect the full complexity of exchange in $\mathrm{Si}$ (see text for further details), they do suggest that high contrast between ground and excited exchange strengths is available.

The excited state Hamiltonian $\mathbf{H}^{\mathrm{e}}$ is

$$
\mathbf{H}^{\mathrm{e}}=\left[\begin{array}{cccccccc}
D_{0} & 0 & 0 & 0 & 0 & 0 & 0 & 0 \\
0 & D_{1} & 2 J_{\mathrm{B}} & 0 & 2 J_{\mathrm{A}} & 0 & 0 & 0 \\
0 & 2 J_{\mathrm{B}} & D_{2} & 0 & 0 & 0 & 0 & 0 \\
0 & 0 & 0 & D_{3} & 0 & 0 & 2 J_{\mathrm{A}} & 0 \\
0 & 2 J_{\mathrm{A}} & 0 & 0 & D_{4} & 0 & 0 & 0 \\
0 & 0 & 0 & 0 & 0 & D_{5} & 2 J_{\mathrm{B}} & 0 \\
0 & 0 & 0 & 2 J_{\mathrm{A}} & 0 & 2 J_{\mathrm{B}} & D_{6} & 0 \\
0 & 0 & 0 & 0 & 0 & 0 & 0 & D_{7}
\end{array}\right]
$$

with diagonal terms $D_{r}$ given by

$$
D_{r}=(-1)^{i} B_{\mathrm{A}}+(-1)^{j} B_{\mathrm{B}}+(-1)^{k} B_{\mathrm{C}}+(-1)^{[i+k]} J_{\mathrm{A}}+(-1)^{[j+k]} J_{\mathrm{B}}
$$

where, once again, $\{i j k\}$ is the binary representation of $r$. 


\subsection{The laser excitation}

Finally, the off-diagonal coupling $\mathbf{L}(t)$ is due to the laser. Since the laser does not produce spin flips the coupling has the form

$$
\mathbf{L}(t)=V(t) \cos (\omega t+\phi) \mathbf{I}
$$

where $\mathbf{I}$ is the $8 \times 8$ unit matrix and

$$
V(t)=\mathbf{d} \cdot \mathbf{E}(t)
$$

where $\mathbf{d}=e\left\langle\psi_{\mathrm{C}}^{\mathrm{e}}|\mathbf{r}| \psi_{\mathrm{C}}^{\mathrm{g}}\right\rangle$ is the dipole matrix element for the $|\mathrm{g}\rangle \rightarrow|\mathrm{e}\rangle$ transition and $\mathbf{E}(t)$ is the pulsed laser's electric field. Here $e$ is the charge on the electron, $\psi_{\mathrm{C}}^{\mathrm{g}}(\mathbf{r}), \psi_{\mathrm{C}}^{\mathrm{e}}(\mathbf{r})$ are the initial and final spatial wavefunctions for particle $C$, whose spatial coordinate is $\mathbf{r}$. In the operation of a quantum gate, a sequence of pulses $\mathbf{L}(t)$ will be chosen to manipulate the entanglement of the qubits $\mathrm{A}$ and $\mathrm{B}$.

\subsection{The control of the qubits}

The spectrum associated with the Hamiltonian in equation (2) has two characteristic energies. The first is $\epsilon$, somewhat less than $1 \mathrm{eV}$, the excitation energy of the excited electronic state. Secondly, the ground and excited (electronic) states are each split into octets, with a very much smaller characteristic energy scale determined by the exchange splitting and magnetic field. This fine structure spin splitting is typically GHz. Now, a pulsed laser, resonantly tuned to the electronic excitation energy $\epsilon$ and with a pulse transform bandwidth much greater than the fine structure splitting, will simply interchange the ground and excited state wavefunctions if the 'pulse area' $\int V(t) \mathrm{d} t=\pi$. If we assume that the system starts in its ground electronic state (but in an arbitrary spin state) then a pair of laser pulses, separated by a time $T$ will cause the system to propagate in its excited electronic state for a time $T$. If, furthermore, we have $T \gg \tau$, where $\tau$ is the laser pulse duration, we may separate the excitation and de-excitation processes from the free propagation between pulses and write for the full time-development operator

$U=\left[\begin{array}{cc}0 & -\mathrm{ie}^{\mathrm{i} \phi} \mathbf{I} \\ -\mathrm{ie}^{-\mathrm{i} \phi} \mathbf{I} & 0\end{array}\right]\left[\begin{array}{cc}U^{\mathrm{g}} & 0 \\ 0 & U^{\mathrm{e}}\end{array}\right]\left[\begin{array}{cc}0 & -\mathrm{ie}^{\mathrm{i} \phi} \mathbf{I} \\ -\mathrm{ie}^{-\mathrm{i} \phi} \mathbf{I} & 0\end{array}\right]=-\left[\begin{array}{cc}U^{\mathrm{e}} & 0 \\ 0 & U^{\mathrm{g}}\end{array}\right]$

where $\mathbf{I}$ is the $8 \times 8$ unit matrix corresponding to the laser excitation and de-excitation, and

$$
U^{\mathrm{g}}=\exp -\mathrm{i}^{\mathrm{g}} T ; \quad U^{\mathrm{e}}=\exp -\mathrm{i}^{\mathrm{e}} T
$$

are the time-development operators for free propagation in the ground and excited states. This effectively allows us to apply $\mathbf{H}^{\mathrm{e}}$ (equation (11)) to the ground state, for a controllable time $T$. Thus, the laser excitation allows us to combine two energy scales: the excitation energy $\epsilon=\hbar \omega$ and the much smaller spin splitting $\sim \hbar / \tau$.

\section{The idealized gate}

We must now consider how this control scheme can be used to make quantum gates.

\subsection{The external parameters-disentangling $C$}

The time development determined by $\mathbf{H}^{\mathrm{e}}$ is discussed in detail in the appendix. To show the essential features we take two steps. First we make some assumptions about the physical parameters which, while not essential, are likely to be reasonable first approximations to experimental realizations of our model. Secondly, we find it convenient to re-order the basis 
states, so that the separation between the control and qubit electrons is easier to see. We use the subscript 2 to indicate that the time-development operators are calculated in this re-ordered basis.

The physical system is defined by a number of parameters $J_{\mathrm{A}}, J_{\mathrm{B}}, B_{\mathrm{A}}, B_{\mathrm{B}}, B_{\mathrm{C}}$, etc, and a propagation time $T$. We shall assume that

$$
\begin{array}{ll}
B_{\mathrm{A}}=B_{\mathrm{B}}=B & \text { say } \\
J_{\mathrm{A}}=J_{\mathrm{B}}=J & \text { say }
\end{array}
$$

(which is what one would expect if particles A and B are identical to each other). For reasons which will become apparent below, we pick two integers $M$ and $N$, and restrict the propagation time $T$ and the magnetic field strength $B$ so that

$$
T=M \pi / \sqrt{\left(B-B_{\mathrm{C}}-J\right)^{2}+8 J^{2}}=N \pi / \sqrt{\left(-B+B_{\mathrm{C}}-J\right)^{2}+8 J^{2}}
$$

which implies that the field strengths $B, B_{\mathrm{C}}$ and Heisenberg coupling $J$ are related by ${ }^{2}$

$$
B-B_{\mathrm{C}}=f J
$$

with

$$
f=-\frac{M^{2}+N^{2}}{M^{2}-N^{2}} \pm \sqrt{\left(\frac{M^{2}+N^{2}}{M^{2}-N^{2}}\right)^{2}-9}
$$

The only restrictions on $M$ and $N$ are that $T$ must be positive and $f$ must be real. The fact that such restrictions are necessary limits the range of two-qubit gates available to us. Nevertheless, we shall show that this limited set of gates includes those which are needed for a universal quantum computer; indeed we show that we can make either a phase gate or a root swap gate to high accuracy.

\subsection{The gate time-development operator}

We now re-order the basis states as

$$
\left[[|0\rangle,|2\rangle,|4\rangle,|6\rangle,|1\rangle,|3\rangle,|5\rangle,|7\rangle]_{\mathrm{g}}[|0\rangle,|2\rangle,|4\rangle,|6\rangle,|1\rangle,|3\rangle,|5\rangle,|7\rangle]_{\mathrm{e}}\right]^{\mathrm{T}}
$$

where, for brevity, we have replaced each spin index $|i j k\rangle$ by its decimal equivalent.

With these assumptions, we can calculate the time-development operator for the excited state. It is

$$
U_{2}^{\mathrm{e}}=\left[\begin{array}{cc}
U_{+}(M, N) & 0 \\
0 & U_{-}(M, N)
\end{array}\right]
$$

with $U_{ \pm}(M, N)$ the $4 \times 4$ unitary matrices

$$
\begin{aligned}
& U_{+}(M, N)=\mathrm{e}^{\mathrm{i}(J-B) T} \\
& \times\left[\begin{array}{cccc}
\mathrm{e}^{-\mathrm{i}[(3-f) J+2 B] T} & 0 & 0 & 0 \\
0 & {\left[(-1)^{M}+\mathrm{e}^{-\mathrm{i}(1-f) J T}\right] / 2} & {\left[(-1)^{M}-\mathrm{e}^{-\mathrm{i}(1-f) J T}\right] / 2} & 0 \\
0 & {\left[(-1)^{M}-\mathrm{e}^{-\mathrm{i}(1-f) J T}\right] / 2} & {\left[(-1)^{M}+\mathrm{e}^{-\mathrm{i}(1-f) J T}\right] / 2} & 0 \\
0 & 0 & 0 & \mathrm{e}^{2 \mathrm{i} B T}(-1)^{N}
\end{array}\right]
\end{aligned}
$$

2 Remembering the definition of $B, B_{\mathrm{C}}$ (equations (5), (14)), we see that, provided $\mu_{\mathrm{A}} \neq \mu_{\mathrm{C}}$, equation (16) can be satisfied by suitably choosing the external magnetic field $\mathbf{B}$. 
and

$$
\begin{aligned}
& U_{-}(M, N)=\mathrm{e}^{\mathrm{i}(J+B) T} \\
& \times\left[\begin{array}{cccc}
\mathrm{e}^{-2 \mathrm{i} B T}(-1)^{M} & 0 & 0 & 0 \\
0 & {\left[(-1)^{N}+\mathrm{e}^{-\mathrm{i}(1+f) J T}\right] / 2} & {\left[(-1)^{N}-\mathrm{e}^{-\mathrm{i}(1+f) J T}\right] / 2} & 0 \\
0 & {\left[(-1)^{N}-\mathrm{e}^{-\mathrm{i}(1+f) J T}\right] / 2} & {\left[(-1)^{N}+\mathrm{e}^{-\mathrm{i}(1+f) J T}\right] / 2} & 0 \\
0 & 0 & 0 & \mathrm{e}^{-\mathrm{i}[(3+f) J-2 B] T}
\end{array}\right] .
\end{aligned}
$$

$U_{+}$describes the evolution of the system when the control spin, $\mathrm{C}$, is initially placed in the excited state with spin up, and $U_{-}$describes the evolution when the spin is down. The blockdiagonal form of $U_{2}^{\mathrm{e}}$ is a consequence of conditions (15) and (17), and is important because it ensures that the control spin always returns to its original state at the end of the gate operation. This means that the control spin is not finally entangled with the qubit spins, and hence does not cause decoherence of the qubit system.

Now consider the operation of an idealized gate. The qubits are encoded in the spin states of $\mathrm{A}$ and $\mathrm{B}$. Thus, the new ordering of the basis states corresponds to the four qubit states and $\mathrm{C}$ spin up, followed by the same four qubit states and $\mathrm{C}$ spin down. Thus, if we insist that particle $\mathrm{C}$ is initially in its spin up state, then after the application of the laser pulses, particle $\mathrm{C}$ will still be spin up in its ground state, but the qubit states will have experienced the time-development operator $U_{+}(M, N)$. Similarly if $\mathrm{C}$ is started in the spin down state the qubits experience the time-development operator $U_{-}(M, N)$. Thus, the effective qubit time-development operator is either $U_{+}$or $U_{-}$; in what follows we shall, generally, assume it to be $U_{+}$, and we shall see that it does indeed produce entanglement, and, for special values of $M$ and $N$ that it produces either a phase gate, which is locally equivalent to a CNOT gate, or a root swap gate.

\section{Two-qubit gates and entanglement}

We now discuss the quantum computational aspects of gates which are easily made by this scheme. This requires some more detailed exploration of the entanglement our protocol can produce.

\subsection{General features of entanglement}

Our quantum information is encoded in the spin states of A and B. Furthermore, as we have described in the previous section, the paired laser pulses return $\mathrm{C}$ to its ground state in the spin state in which it started, which we choose to be $|0\rangle_{\mathrm{gC}}$. Therefore we confine our attention to wavefunctions of the form

$$
\Psi=\psi_{\mathrm{A}} \psi_{\mathrm{B}} \psi_{\mathrm{C}}^{\mathrm{g}}\left[c_{0}|00\rangle+c_{1}|01\rangle+c_{2}|10\rangle+c_{3}|11\rangle\right]|0\rangle_{\mathrm{gC}}
$$

where the two spin states in the brackets $[\cdots]$ are states of $\mathrm{A}$ and $\mathrm{B}$, and $|0\rangle_{\mathrm{gC}}$ is the spin up state of (the ground state of) $\mathrm{C}$. Although the spin of $\mathrm{C}$ has an important role in the excited state, the conditions expressed in equations (15) and (17) guarantee that it only acts as a spectator when $\mathrm{C}$ has returned to its ground electronic state. All the quantum information for the two-qubit gate is encoded in $\mathbf{c}$, the column vector

$$
\mathbf{c}=\left[c_{0}, c_{1}, c_{2}, c_{3}\right]^{\mathrm{T}}
$$

on which the time-development operator $U_{+}(M, N)$ acts. The form of the time-development operator, that is, the values of $M$ and $N$, determines the type of gate.

We shall have to distinguish between local operations, which can be decomposed into a series of manipulations on A and B separately, and two-qubit operations, which manipulate 
the joint state of A and B. Physically, the former can be performed by A-gates (acting on single qubits) alone. Generally, single-spin manipulations of this type will be performed using already-established methods that are different to what we describe here (Charnock and Kennedy 2001, Jelezko et al 2002).

The scheme we describe is supposed to produce a so-called J-gate, which can perform two-qubit operations. These depend on the state of both spins. A characteristic of such gates is that, in contrast to A-gates, they can produce entanglement between the spins of A and B from a state which is initially unentangled (Wootters 1998). Such operations are required to perform the universal quantum logical operations which give quantum computing its power.

Thus, in our scheme, a general quantum gate will consist of a combination of A- and J-gates, and the gate will be represented by a time-development operator of the form

$$
U_{\text {gate }}=L_{\mathrm{f}} U_{+}(M, N) L_{\mathrm{i}}
$$

where $L_{\mathrm{i}}$ and $L_{\mathrm{f}}$ represent local operations, performed with A-gates and $U_{+}(M, N)$ provides the J-gate. We stress that this paper is not concerned with the implementation of the local gates $L_{\mathrm{i}}$ and $L_{\mathrm{f}}$. Indeed, it is known that the entanglement produced by $U_{\text {gate }}$ does not depend on the form of $L_{\mathrm{i}}$ and $L_{\mathrm{f}}$. Gates with the same $U_{+}(M, N)$, but different $L_{\mathrm{i}}$ and $L_{\mathrm{f}}$, are said to be locally equivalent. Although they may do different things, they can be made equal by using only A-gates.

Makhlin (2000) has considered how to characterize locally equivalent gates of two qubits. First, we transform to what he calls the Bell basis

$$
\left[\frac{1}{\sqrt{2}}(|00\rangle+|11\rangle), \frac{\mathrm{i}}{\sqrt{2}}(|01\rangle+|10\rangle), \frac{1}{\sqrt{2}}(|01\rangle-|10\rangle), \frac{\mathrm{i}}{\sqrt{2}}(|00\rangle-|11\rangle)\right]
$$

which we identify with the subscript $\mathcal{B}$. We have

$$
\mathbf{c}_{\mathcal{B}}=Q \mathbf{c}
$$

and

$$
U_{+\mathcal{B}}=Q^{\dagger} U_{+} Q
$$

with

$$
Q=\frac{1}{\sqrt{2}}\left[\begin{array}{cccc}
1 & 0 & 0 & \mathrm{i} \\
0 & \mathrm{i} & 1 & 0 \\
0 & \mathrm{i} & -1 & 0 \\
1 & 0 & 0 & -\mathrm{i}
\end{array}\right]
$$

He shows that, given a gate time-development operator $U$ in the standard basis, one can calculate

$$
m=U_{\mathcal{B}}^{\mathrm{T}} U_{\mathcal{B}}
$$

and

$$
n=\operatorname{det} U
$$

and then find

$$
G_{1}=\operatorname{tr}^{2}(m) / 16 n
$$

and

$$
G_{2}=\left(\operatorname{tr}^{2}(m)-\operatorname{tr}\left(m^{2}\right)\right) / 4 n .
$$

These two quantities between them uniquely specify the eigenvalues of $m$. Two gate time-development operators $U_{x}$ and $U_{y}$ are then equivalent to one another within local transformations if and only if they have the same values of $G_{1}$ and $G_{2}$. 
We may now apply these ideas to $U_{+}(M, N)$. We get

$$
G_{1}(M, N)=(-1)^{(M+N)}\left[\mathrm{e}^{-\mathrm{i} J T}+(-1)^{N} \mathrm{e}^{\mathrm{i} J T} \cos (1-f) J T\right]^{2} / 4
$$

and

$$
G_{2}(M, N)=(-1)^{(M+N)}\left[\cos 2 J T+2(-1)^{N} \cos (1-f) J T\right]
$$

where equations (17) and (15) must be used for $f$ and $T$.

\subsection{The phase gate, the 'root swap' gate}

The purpose of this work is to consider the use of excited states to control two-qubit gates. To avoid the (for our purposes) unnecessary complications introduced by local one-qubit gates we focus on two well known universal gates- the phase gate and the so-called 'root swap' gate. The phase gate has the unitary transform

$$
U_{\text {phase }}=\left[\begin{array}{cccc}
1 & 0 & 0 & 0 \\
0 & 1 & 0 & 0 \\
0 & 0 & 1 & 0 \\
0 & 0 & 0 & -1
\end{array}\right]
$$

so that

$$
\begin{aligned}
& G_{1}^{\text {phase }}=0 \\
& G_{2}^{\text {phase }}=1
\end{aligned}
$$

whereas the root swap gate we shall consider is of the form

$$
U_{\mathrm{rs}}=\left[\begin{array}{cccc}
1 & 0 & 0 & 0 \\
0 & (1-\mathrm{i}) / 2 & -(1+\mathrm{i}) / 2 & 0 \\
0 & -(1+\mathrm{i}) / 2 & (1-\mathrm{i}) / 2 & 0 \\
0 & 0 & 0 & -1
\end{array}\right]
$$

so that

$$
\begin{aligned}
G_{1}^{\mathrm{rs}} & =\frac{-\mathrm{i}}{4} \\
G_{2}^{\mathrm{rs}} & =0 .
\end{aligned}
$$

If values of $M$ and $N$ can be found such that

$$
\begin{aligned}
& G_{1}^{U_{+}(M, N)}=G_{1}^{\text {gate }}+\delta_{1} \\
& G_{2}^{U_{+}(M, N)}=G_{2}^{\text {gate }}+\delta_{2}
\end{aligned}
$$

(gate $=$ phase, rs) where $\delta_{1}$ and $\delta_{2}$ are 'small', then the corresponding gate time-development operator will be 'near' a time-development operator which is locally equivalent to either a phase gate or a root swap gate. The reason for focusing on these gates rather than the more familiar CNOT gate (Cirac and Zoller 1995), is that, as we shall see, the values of $M, N$ which satisfy equations (34) or (36) actually produce time development operators close to those given in equations (33) or (35) rather than a local equivalent. This enables us to determine the target matrix $U_{W}$ defined below, and therefore the quality of the gate protocol, without unnecessary complications.

We characterize the system by its (entanglement) fidelity, (Nielsen 2002, Fortunato et al 2002) defined as

$$
\mathcal{F}_{+}(M, N)=\left|\frac{1}{4} \operatorname{Tr}\left\{U_{W}^{\dagger} U_{+}(M, N)\right\}\right|^{2}
$$


Table 1. Some parameter sets which produce two-qubit gates and their errors. Each set is an extreme: amongst those we have considered set 1 has the smallest value of $\Delta_{+}$and set 3 the smallest value of $J T$. Sets 2 and 4 have the smallest values of $\left(B_{\mathrm{A}}-B_{\mathrm{C}}\right) / J$ for a phase gate and root swap gate, respectively.

\begin{tabular}{llrrcccc}
\hline Set & Type & \multicolumn{1}{c}{$M$} & $N$ & $\left(B_{\mathrm{A}}-B_{\mathrm{C}}\right) / J$ & \multicolumn{1}{l}{$J T$} & $\sqrt{\delta_{1}^{2}+\delta_{2}^{2}}$ & $\Delta_{+}(M, N)$ \\
\hline 1 & Phase & 1584 & 2177 & 4.5 & 1105.84 & $1.6 \times 10^{-6}$ & $4.8 \times 10^{-7}$ \\
2 & Phase & 1534 & 1444 & -0.274 & 1553.522 & $3.0 \times 10^{-5}$ & $3.1 \times 10^{-5}$ \\
3 & $\sqrt{\text { Swap }}$ & 563 & 618 & 21.093 & 87.169 & $1.8 \times 10^{-4}$ & $1.6 \times 10^{-4}$ \\
4 & $\sqrt{\text { Swap }}$ & 1631 & 1479 & -0.449 & 1612.405 & $4.7 \times 10^{-4}$ & $2.9 \times 10^{-3}$ \\
\hline
\end{tabular}

where $U_{W}$ is the time dependent operator to which $U_{+}(M, N)$ itself is close (equations (33) or (35)).

In fact, it is more useful to use

$$
\Delta_{+}(M, N)=1-\mathcal{F}_{+}(M, N)
$$

so that values close to zero are desirable. Some values of $\Delta_{+}(M, N)$ are given in table 1 . It is clear that the ideal gate protocol delivers adequate fidelities. However, we stress that the values of $\Delta_{+}(M, N)$ given in table 1 are idealizations. They represent the closest that this protocol can come to producing either a phase gate or root swap gate while still satisfying equation (15). We must now consider how a more realistic protocol will operate.

\section{A 'realistic' gate}

We have made many idealizations in constructing table 1 . In this section we consider the laser excitation in more detail and also estimate the effect of decoherence in the operation of the gates.

Even in the absence of decoherence there are two idealizations hidden in equation (12). First, it is assumed that the laser pulse transform bandwidth is large enough to cover all the spin components of the excited state, and secondly that the laser pulse lengths $\tau$ are negligible in comparison with $T$, the interval between them. For the parameters discussed here the first criterion is several orders of magnitude stricter than the second. Typical splittings in the excited state are of the order of $|1-f| J$, so that we need

$$
J \tau \ll 1 /|1-f|
$$

to ensure that all the components are properly excited, whereas the pulse will be short in comparison to the gate protocol duration if

$$
J \tau \ll J T .
$$

Typically, $1 /|1-f| \sim 1$, whereas $J T \sim 1000$.

In figure 3 we show calculations of fidelities as a function of the laser pulse duration. Typical values for the ideal case $(\tau \rightarrow 0)$ are

$$
\Delta_{+} \sim 3 \times 10^{-7}-10^{-4}
$$

so we can see that, although these values are not achieved for real laser excitation, there is a range of laser parameters for which usefully accurate gates can be made. Notice that although parameter sets 3 and 4 have poorer ideal fidelities, they can tolerate longer pulse lengths before their fidelities become unacceptable. This is because the small magnetic field required in these cases implies that there is a smaller energy spread of upper state sublevels, and therefore a longer laser pulse can still excite them all. 


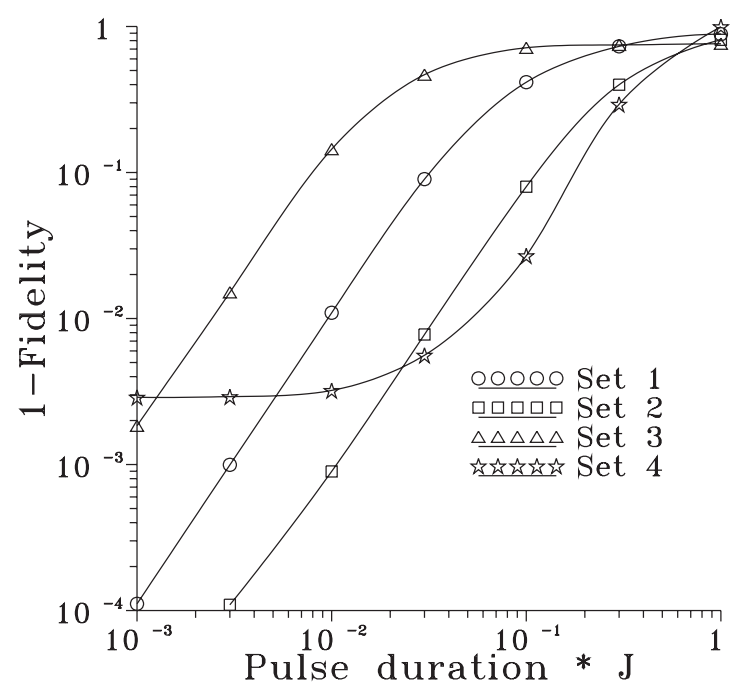

Figure 3. The gate error as a function of laser pulse duration for the four parameter sets in table 1.

Table 2. Typical values required for a realistic gate. We show values of the laser pulse duration and magnetic field required to make a phase gate as a function of the $\sigma \cdot \sigma$ interaction strength for three cases, all chosen so that $J \tau \sim 0.01-0.1$. (For orientation, the exchange interaction between a 1s and 3d electron in He has a strength of $0.35 \mathrm{meV}$ (Bethe and Salpeter 1957).) Typically, a visible or near infra-red laser with a fluence of $\sim 0.5 \mathrm{~J} \mathrm{~m}^{-2}$ per pulse will be needed. Averaging over the temporal and spatial distribution of the pulses, and noting that the gate protocol requires two short pulses separated by a long time interval, and, furthermore, the qubits themselves are not densely distributed, we estimate that this implies a mean power density of $\sim 10 \mathrm{~W} \mathrm{~m}^{-2}$ in case 1 . It should be noted that, in ideal operation, all energy absorbed would be re-emitted as photons at the end of gate operation.

\begin{tabular}{lllll}
\hline Case & $J$ & $\tau$ & $B(\mathrm{~T})$ & $t_{\text {sep }}(\mathrm{ns})$ \\
\hline 1 & $6.6 \mu \mathrm{V}=1.6 \mathrm{GHz}$ & $1-10 \mathrm{ps}$ & $0.01-1$ & $10-100$ \\
2 & $66 \mu \mathrm{V}=16 \mathrm{GHz}$ & $0.1-1 \mathrm{ps}$ & $0.1-10$ & $1-10$ \\
3 & $0.66 \mathrm{mV}=160 \mathrm{GHz}$ & $10-100 \mathrm{fs}$ & $1-100$ & $0.1-1$ \\
\hline
\end{tabular}

As can be seen from figure 3, a value of $J \tau<\sim 0.01-0.1$ is required for a 'small' value of $\Delta_{+}$. This gives a relationship between the laser pulse duration and $J$, the strength of the $\sigma \cdot \sigma$ interaction which drives the gate. This in turn determines the order of magnitude of the magnetic field required to produce the phase gate (see equation (17)). Table 2 shows typical parameters for various realistic values of $J$. Case 1 does not seem to be beyond present-day technology, case 2 is at the limit of what might be possible, but case 3 seems inaccessible.

Finally, we consider the effect of decoherence on the gate. As noted by Stoneham et al (2003) there are several possible sources of decoherence. One is the spontaneous decay of the excited state, which (as remarked earlier) we expect to occur on a microsecond timescale. Another is spin-lattice relaxation; this varies rapidly with temperature. For Si:Bi the groundstate spin-lattice lifetime is longer than a microsecond for temperatures below about $30 \mathrm{~K}$ (Castner 1962), but for other defect systems where the energy splittings exceed the largest phonon energy the situation may be much better.

The ground-state relaxation is particularly critical because it acts even while the gate is in the 'off' state. In order to estimate the decoherence rate tolerable in the 'on' state, we use a 


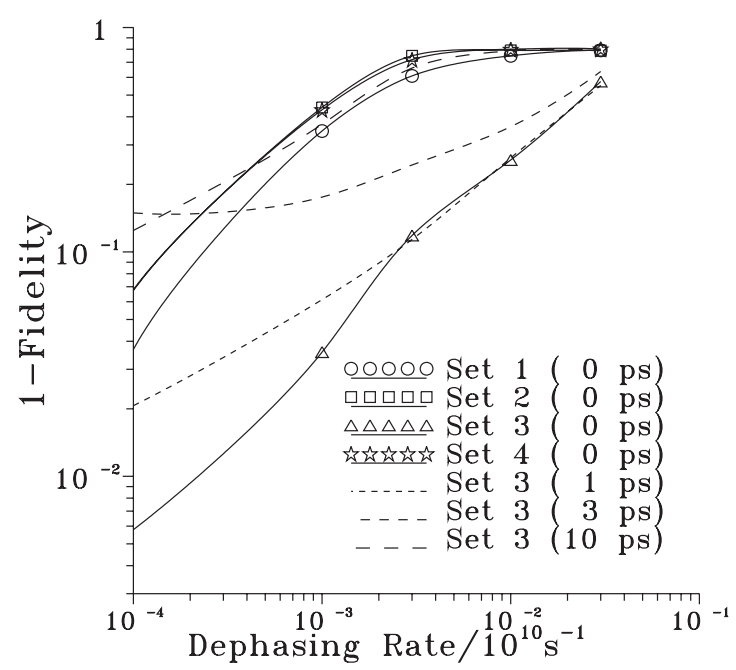

Figure 4. The gate error as a function of decoherence rate. Those labelled 0 ps show how the fidelity falls off as a function of decoherence rate for the four parameter sets, on the assumption that the laser pulse length tends to zero. Clearly parameter set 3, which has the shortest gate duration, performs well here. The dashed curves show how the fidelity behaves for this case, but with more realistic laser pulse lengths.

very simple model of dephasing based on a random telegraph signal. We assume that phase jumps of $\pi$ occur in the upper state wavefunction, randomly at a rate $\Gamma$, and assign each of these phase jumps to a component of the excited state wavefunction chosen at random (Greenland 2003); this corresponds to a $\mathrm{T}_{2}$-type process. We use standard unravelling techniques (Mølmer et al 1993) to calculate observables. We expect that this model will exhibit the main features of most decoherence processes.

We focus on case 1 in table 2 and consider all four parameter sets. In figure 4 we show how the fidelity falls as the decoherence rate rises. Most of these calculations are done with an artificially short laser pulse in order to expose the degradation of fidelity with collision rate. It is clear that the much shorter gate protocol time associated with parameter set 3 ( $\sim 9 \mathrm{~ns}$, rather than the 100-150 ns required in the other cases) is advantageous, and outweighs the rapid degradation in fidelity with laser pulse duration noted above for this parameter set. Figure 4 shows that dephasing rates of up to $10^{7} \mathrm{~s}^{-1}$ (or even up to $10^{8} \mathrm{~s}^{-1}$ ) are tolerable for demonstration purposes. We can therefore accept relaxation times one to two orders of magnitude faster than those known for the ground state of $\mathrm{Si}: \mathrm{Bi}$ at $30 \mathrm{~K}$.

\section{Summary}

In this paper, as in (Stoneham et al 2003), we have described a solid-state two-qubit quantum gate which, in contrast to many other designs, separates the storage and interaction aspects of the gate by using an excited control particle to produce the qubit-qubit interaction necessary for entanglement. Here we analyse the important requirement that the control particle should remain unentangled with the qubits after the gate protocol is over; this restricts the effective two-qubit gates we can make, through equations (15) and (17)_-all the useful gates are defined by two integers $M$ and $N$. Values of these integers can be found which give either phase or root swap gates. Implementation of these gates with pulsed laser excitation of defects in Si is within 
the scope of present-day technology, and preliminary exploration suggests that decoherence during the gate operation, while important, is likely to be at a tolerable level.

We stress that in this paper we have been concerned to show what is possible with a very simple implementation of an excitation controlled two-qubit gate, and many extensions of the idea are possible. Some will be described elsewhere. The fact that 'classic' two-qubit gates which can be made without undue technological effort can be found in a preliminary exploration of the parameter space available to us suggests that much more can be done.

\section{Acknowledgments}

We are grateful to Professors G Aeppli, P Bayvel, I Boyd, Drs A H Harker and C Renner for valuable discussions. This work was done under EPSRC grant GR/S23506.

\section{Appendix}

Calculation of the time development operator is simplified by permuting the basis states so that $|3\rangle$ and $|4\rangle$ interchange their positions. With this permutation the excited state Hamiltonian (8) becomes

$$
\mathbf{H}_{1}^{\mathrm{e}}=\left[\begin{array}{cccccccc}
D_{0} & 0 & 0 & 0 & 0 & 0 & 0 & 0 \\
0 & D_{1} & 2 J_{\mathrm{B}} & 2 J_{\mathrm{A}} & 0 & 0 & 0 & 0 \\
0 & 2 J_{\mathrm{B}} & D_{2} & 0 & 0 & 0 & 0 & 0 \\
0 & 2 J_{\mathrm{A}} & 0 & D_{4} & 0 & 0 & & 0 \\
0 & 0 & 0 & 0 & D_{3} & 0 & 2 J_{\mathrm{A}} & 0 \\
0 & 0 & 0 & 0 & 0 & D_{5} & 2 J_{\mathrm{B}} & 0 \\
0 & 0 & 0 & 0 & 2 J_{\mathrm{A}} & 2 J_{\mathrm{B}} & D_{6} & 0 \\
0 & 0 & 0 & 0 & 0 & 0 & 0 & D_{7}
\end{array}\right]
$$

which is in a convenient block diagonal form. Now if

$$
\begin{aligned}
& D_{2}=D_{4} \\
& D_{3}=D_{5}
\end{aligned}
$$

then the $3 \times 3$ sub-matrices can be diagonalized easily, and the time development operator written in closed form. Although this is not the only circumstance in which this is possible, the conditions (42) imply

$$
\begin{array}{ll}
B_{\mathrm{A}}=B_{\mathrm{B}}=B & \text { say } \\
J_{\mathrm{A}}=J_{\mathrm{B}}=J & \text { say }
\end{array}
$$

which is what one would expect if particles A and B are identical to each other.

The time-development operator is then

$$
U_{1}^{\mathrm{e}}(t)=\left[\begin{array}{cccc}
\exp \left(-\mathrm{i} D_{0} t\right) & 0 & 0 & 0 \\
0 & R(t) & 0 & 0 \\
0 & 0 & S(t) & 0 \\
0 & 0 & 0 & \exp \left(-\mathrm{i} D_{7} t\right)
\end{array}\right]
$$

where $R$ can be written

$$
R=\mathrm{e}^{\mathrm{i}(J-B) t}\left[\begin{array}{ccc}
\mathrm{c}-\mathrm{i} u / p \mathrm{~s} & -2 \mathrm{i} J / p \mathrm{~s} & -2 \mathrm{i} J / p \mathrm{~s} \\
-2 \mathrm{i} J / p \mathrm{~s} & {\left[\mathrm{c}+\mathrm{i} u / p \mathrm{~s}+\mathrm{e}^{\mathrm{i} u t}\right] / 2} & {\left[\mathrm{c}+\mathrm{i} u / p \mathrm{~s}-\mathrm{e}^{\mathrm{i} u t}\right] / 2} \\
-2 \mathrm{i} J / p \mathrm{~s} & {\left[\mathrm{c}+\mathrm{i} u / p \mathrm{~s}-\mathrm{e}^{\mathrm{i} u t}\right] / 2} & {\left[\mathrm{c}+\mathrm{i} u / p \mathrm{~s}+\mathrm{e}^{\mathrm{i} u t}\right] / 2}
\end{array}\right]
$$


with

$$
\begin{aligned}
& u=B-B_{\mathrm{C}}-J \\
& p=\sqrt{u^{2}+8 J^{2}} \\
& \mathrm{c}=\cos p t \\
& \mathrm{~s}=\sin p t
\end{aligned}
$$

and $S$ is

$S=\mathrm{e}^{\mathrm{i}(J+B) t}\left[\begin{array}{ccc}{\left[\overline{\mathrm{c}}+\mathrm{i} v / q \overline{\mathrm{s}}+\mathrm{e}^{\mathrm{i} v t}\right] / 2} & {\left[\overline{\mathrm{c}}+\mathrm{i} v / q \overline{\mathrm{s}}-\mathrm{e}^{\mathrm{i} v t}\right] / 2} & -2 \mathrm{i} J / q \overline{\mathrm{s}} \\ {\left[\overline{\mathrm{c}}+\mathrm{i} v / q \overline{\mathrm{s}}-\mathrm{e}^{\mathrm{i} v t}\right] / 2} & {\left[\overline{\mathrm{c}}+\mathrm{i} v / q \overline{\mathrm{s}}+\mathrm{e}^{\mathrm{i} v t}\right] / 2} & -2 \mathrm{i} J / q \overline{\mathrm{s}} \\ -2 \mathrm{i} J / q \overline{\mathrm{s}} & -2 \mathrm{i} J / q \overline{\mathrm{s}} & \overline{\mathrm{c}}-\mathrm{i} v / q \overline{\mathrm{s}}\end{array}\right]$

with

$$
\begin{aligned}
& v=-B+B_{\mathrm{C}}-J \\
& q=\sqrt{v^{2}+8 J^{2}} \\
& \overline{\mathrm{c}}=\cos q t \\
& \overline{\mathrm{s}}=\sin q t .
\end{aligned}
$$

We now perform a final permutation to bring the basis into the order

$$
\left[[|0\rangle,|2\rangle,|4\rangle,|6\rangle,|1\rangle,|3\rangle,|5\rangle,|7\rangle]_{\mathrm{g}}[|0\rangle,|2\rangle,|4\rangle,|6\rangle,|1\rangle,|3\rangle,|5\rangle,|7\rangle]_{\mathrm{e}}\right]^{\mathrm{T}}
$$

and the corresponding time-development operator in the excited state is

$$
U_{2}^{\mathrm{e}}=\left[\begin{array}{cccccccc}
\exp \left(-\mathrm{i} D_{0} t\right) & 0 & 0 & 0 & 0 & 0 & 0 & 0 \\
0 & R_{22} & R_{32} & 0 & R_{21} & 0 & 0 & 0 \\
0 & R_{23} & R_{33} & 0 & R_{31} & 0 & 0 & 0 \\
0 & 0 & 0 & S_{33} & 0 & S_{31} & S_{32} & 0 \\
0 & R_{12} & R_{13} & 0 & R_{11} & 0 & 0 & 0 \\
0 & 0 & 0 & S_{13} & 0 & S_{11} & S_{12} & 0 \\
0 & 0 & 0 & S_{23} & 0 & S_{21} & S_{22} & 0 \\
0 & 0 & 0 & 0 & 0 & 0 & 0 & \exp \left(-\mathrm{i} D_{7} t\right)
\end{array}\right] .
$$

Now, let us choose magnetic fields $B$ and $B_{\mathrm{C}}$, interaction strength $J$ and total time $T$ so that

$$
p T=M \pi
$$

and

$$
q T=N \pi
$$

This will be possible if the magnetic and Heisenberg interaction strengths are related by $B-B_{\mathrm{C}}=f J$ with $f$ given by

$$
f=-\frac{M^{2}+N^{2}}{M^{2}-N^{2}} \pm \sqrt{\left(\frac{M^{2}+N^{2}}{M^{2}-N^{2}}\right)^{2}-9}
$$

and under these conditions

$$
R_{12}=R_{13}=R_{21}=R_{31}=S_{13}=S_{23}=S_{31}=S_{32}=0
$$

so that equation (49) becomes

$$
U_{2}^{\mathrm{e}}=\left[\begin{array}{cc}
U_{+}(M, N) & 0 \\
0 & U_{-}(M, N)
\end{array}\right]
$$


with $U_{ \pm}(M, N)$ the $4 \times 4$ time-development operators

$$
\begin{aligned}
& U_{+}(M, N)=\mathrm{e}^{\mathrm{i}(J-B) T} \\
& \times\left[\begin{array}{cccc}
\mathrm{e}^{-\mathrm{i}[(3-f) J+2 B] T} & 0 & 0 & 0 \\
0 & {\left[(-1)^{M}+\mathrm{e}^{-\mathrm{i}(1-f) J T}\right] / 2} & {\left[(-1)^{M}-\mathrm{e}^{-\mathrm{i}(1-f) J T}\right] / 2} & 0 \\
0 & {\left[(-1)^{M}-\mathrm{e}^{-\mathrm{i}(1-f) J T}\right] / 2} & {\left[(-1)^{M}+\mathrm{e}^{-\mathrm{i}(1-f) J T}\right] / 2} & 0 \\
0 & 0 & 0 & \mathrm{e}^{2 \mathrm{i} B T}(-1)^{N}
\end{array}\right]
\end{aligned}
$$

and

$$
\begin{aligned}
& U_{-}(M, N)=\mathrm{e}^{\mathrm{i}(J+B) T} \\
& \times\left[\begin{array}{cccc}
\mathrm{e}^{-2 \mathrm{i} B T}(-1)^{M} & 0 & 0 & 0 \\
0 & {\left[(-1)^{N}+\mathrm{e}^{-\mathrm{i}(1+f) J T}\right] / 2} & {\left[(-1)^{N}-\mathrm{e}^{-\mathrm{i}(1+f) J T}\right] / 2} & 0 \\
0 & {\left[(-1)^{N}-\mathrm{e}^{-\mathrm{i}(1+f) J T}\right] / 2} & {\left[(-1)^{N}+\mathrm{e}^{-\mathrm{i}(1+f) J T}\right] / 2} & 0 \\
0 & 0 & 0 & \mathrm{e}^{-\mathrm{i}[(3+f) J-2 B] T}
\end{array}\right]
\end{aligned}
$$

which are the desired results.

\section{References}

Alvarez Collado J R, Fernández Rico J, López R, Paniagua M and Ramírez G 1989 Comput. Phys. Commun. 52323 Andres K, Bhatt R N, Goalwin P, Rice T M and Walstedt R E 1981 Phys. Rev. B 24244

Barenco A, Bennett C H, Cleve R, DiVincenzo D P, Margolus N, Shor P, Sleator T, Smolin J A and Weinfurter H 1995 Phys. Rev. A $\mathbf{5 2} 3457$

Beckmann D, Chari A N, Devabhaktuni S and Preskill J 1996 Phys. Rev. A 541034

Bethe H E and Salpeter E E 1957 Quantum Mechanics of One-and Two-Electron Atoms (Berlin: Springer)

Castner T G 1962 Phys. Rev. 13058

Charnock F T and Kennedy T A 2001 Phys. Rev. B 64041201

Cirac J I and Zoller P 1995 Phys. Rev. Lett. 744091

Cullis P R and Marko J R 1970 Phys. Rev. B 1632

DiVincenzo D P 1995 Science 270255

Fernández Rico J, López R, Ema I and Ramírez G 1997 Comput. Phys. Commun. 105216

Fortunato E M, Pravia M A, Boulant N, Teklemariam G, Havel T F and Cory D G 2002 Preprint quant-ph/0202065

Golovach V N and Loss D 2002 Semicond. Sci. Technol. 17355

Greenland P T 2003 Phil. Trans. R. Soc. 361379

Herring C and Flicker M 1964 Phys. Rev. 134 A362

Jelezko F et al 2002 Appl. Phys. Lett. 812160

Jonathan D, Plenio M B and Knight P L 2000 Fast quantum gates for cold atoms Preprint quant-ph/0002092

Koiller B, Hu X and Das Sarma S 2002a Phys. Rev. Lett. 88027903

Koiller B, Hu X and Das Sarma S 2002b Phys. Rev. B 66115201

Loss D and DiVincenzo D P 1998 Phys. Rev. A 57120

Makhlin Yu 2000 Non-local properties of two-qubit gates and mixed states and optimization of quantum computations Preprint quant-phys/0002045

Mølmer K, Castin Y and Dalibard J 1993 J. Opt. Soc. Am. B 10524

Nielsen M 2002 Preprint quant-ph/0205035

Slater J C 1963 Quantum Theory of Molecules and Solids (Electronic Structure of Molecules vol 1) (New York: McGraw-Hill)

Stoneham A M 1975 Theory of Defects in Solids (Oxford: Oxford University Press)

Stoneham A M, Fisher A J and Greenland P T 2003 J. Phys.: Condens. Matter 15 L447

Vedral V and Plenio M B 1998 Prog. Quantum Electron. 221

Wootters W K 1998 Phil. Trans. R. Soc. A 3561717 